PROCEEDINGS OF THE

AMERICAN MATHEMATICAL SOCIETY

Volume 140, Number 1, January 2012, Pages 99-107

S 0002-9939(2011)10968-7

Article electronically published on May 17, 2011

\title{
CONSTRUCTION OF CENTRAL UNITS IN INTEGRAL GROUP RINGS OF FINITE GROUPS
}

\author{
ERIC JESPERS AND M. M. PARMENTER
}

(Communicated by Jonathan I. Hall)

\begin{abstract}
In this paper we give new constructions of central units that generate a subgroup of finite index in the central units of the integral group ring $\mathbb{Z} G$ of a finite group. This is done for a very large class of finite groups $G$, including the abelian-by-supersolvable groups.
\end{abstract}

Let $G$ be a finite group. When $G$ is abelian it is well known (and due to Bass and Milnor; see [1] and 9, Theorem 12.7 and Theorem 13.1]) that the Bass cyclic units of the integral group ring $\mathbb{Z} G$ generate a subgroup of finite index in $\mathcal{Z}(\mathcal{U}(\mathbb{Z} G))$, the group of (central) units of $\mathbb{Z} G$. In [4], it was shown that if $G$ is a nilpotent finite group of nilpotency class $n$, then the group $\left\langle b_{(n)}\right\rangle$ is of finite index in $\mathcal{Z}(\mathcal{U}(\mathbb{Z} G))$. Here $b_{(n)}$ is defined recursively as follows. We denote by $Z_{i}$ the $i$-th centre of $G$. For any $x \in G$ and Bass cyclic unit $b \in \mathbb{Z}\langle x\rangle$, put $b_{(1)}=b$, and, for $2 \leq i \leq n$, put $b_{(i)}=\prod_{g \in Z_{i}} b_{(i-1)}^{g}$, where $\alpha^{g}=g^{-1} \alpha g$ for $\alpha \in \mathbb{Z} G$.

Recently, Ferraz and Simón [2 gave a construction of central units in case $G$ is meta-(cyclic of prime order) that generate a subgroup of finite index. They actually provided an independent set of generators.

In this note we continue these investigations and provide new constructions of central units that generate a subgroup of finite index in the central units of $\mathbb{Z} G$ and this for a very large class of finite groups $G$, including the abelian-by-supersolvable groups. The proof and construction relies on a beautiful paper of Olivieri, del Río and Simón [6] in which they give an explicit construction of the primitive central idempotents of rational group algebras $\mathbb{Q} G$ of the so-called strongly monomial groups (for example, abelian-by-supersolvable groups). Our proof thus uses a completely different method from the one used in 4 for finite nilpotent groups. In Section 1 we will recall the necessary background on this. In Section 2 we prove the main results for strongly monomial groups. In Section 3 we prove a general result that yields generators for central units from subgroups and quotient groups. An application is given for solvable Frobenius groups.

Received by the editors June 3, 2010 and, in revised form, July 20, 2010 and November 10, 2010.

2010 Mathematics Subject Classification. Primary 16S34, 16U60, 16 U70.

Key words and phrases. Group ring, central unit.

The first author was supported in part by Onderzoeksraad of Vrije Universiteit Brussel and Fonds voor Wetenschappelijk Onderzoek (Belgium).

The second author was supported in part by the Natural Sciences and Engineering Research Council of Canada. 
One of the fundamental points in our construction of generators for central units in $\mathbb{Z} G$ is that the constructed units are generic and only make use of the lattice of subgroups of $G$. So the character table is not explicitly used. In [8] generators for a subgroup of finite index were found using the character table of $G$ (and thus the primitive central idempotents of the complex group algebra $\mathbb{C} G$ ) and also using some Galois descent.

\section{Primitive Central idempotents}

Let $G$ be a finite group and $K$ and $H$ subgroups. If $K \unlhd H$, then let $\varepsilon(H, K)=$ $\widehat{K}=\frac{1}{|K|} \sum_{k \in K} k \in \mathbb{Q} K$ if $H=K$, and if $H \neq K$, then let

$$
\varepsilon(H, K)=\prod_{M / K \in \mathcal{M}(H / K)}(\widehat{K}-\widehat{M}),
$$

where $\mathcal{M}(H / K)$ denotes the set of all minimal normal nontrivial subgroups of $H / K$. Clearly $\varepsilon(H, K)$ is an idempotent of $\mathbb{Q} G$. Furthermore, let $e(G, H, K)$ denote the sum of the different $G$-conjugates of $\varepsilon(H, K)$ in $\mathbb{Q} G$; that is, if $T$ is a right transversal of $\operatorname{Cen}_{G}(\varepsilon(H, K))$ in $G$, then

$$
e(G, H, K)=\sum_{t \in T} \varepsilon(H, K)^{t},
$$

where $\operatorname{Cen}_{G}(\varepsilon(H, K))$ denotes the centralizer of $\varepsilon(H, K)$ in $G$. Obviously, $e(G, H, K)$ is a central element of $\mathbb{Q} G$. If the $G$-conjugates of $\varepsilon(H, K)$ are orthogonal, then $e(G, H, K)$ is a central idempotent of $\mathbb{Q} G$.

A Shoda pair of a finite group $G$ is a pair $(H, K)$ of subgroups of $G$ with the properties that $K \unlhd H, H / K$ is cyclic and for every $g \in G \backslash H$ there exists $h \in H$ so that $h^{-1} g^{-1} h g \in H \backslash K$. A strong Shoda pair of $G$ is a Shoda pair $(H, K)$ of $G$ such that $H \unlhd N_{G}(K)$ and the different conjugates of $\varepsilon(H, K)$ are orthogonal.

If $(H, K)$ is a strong Shoda pair, then we also have that $\operatorname{Cen}_{G}(\varepsilon(H, K))=$ $N_{G}(K)$.

Crucial for the proof of our main result is the following proposition.

Proposition 1.1 (Olivieri, del Río and Simón [6]). Let $(H, K)$ be a strong Shoda pair and let $k=[H: K], N=N_{G}(K), n=[G: N], x$ a generator of $H / K$ and $\phi: N / H \rightarrow N$ a left inverse of the projection $N \rightarrow N / H$. Then $\mathbb{Q} G e(G, H, K)$ is a simple ring isomorphic to $M_{n}(\mathbb{Q} N \varepsilon(H, K))=M_{n}\left(\mathbb{Q}\left(\xi_{k}\right) *_{\tau}^{\sigma} N / H\right)$ where the action and twisting defining the crossed product $\mathbb{Q}\left(\xi_{k}\right) *_{\tau}^{\sigma} N / H$ are given by

$$
\begin{array}{rlrl}
\xi_{k}^{\sigma(a)} & =\xi_{k}^{i}, & & \text { if } x^{a}=x^{i}, \\
\tau(a, b) & =\xi_{k}^{j}, & \text { if } \phi(a b)^{-1} \phi(a) \phi(b) H=x^{j},
\end{array}
$$

for $a, b \in N / H$ and integers $i$ and $j$. Note that $\sum_{t \in T}(\mathbb{Q} N \varepsilon(H, K))^{t}$ consists of the diagonal matrices of $M_{n}(\mathbb{Q} N \varepsilon(H, K))$ and that the action of $N / H$ on $\mathbb{Q}\left(\xi_{k}\right)=$ $\mathbb{Q} H \varepsilon(H, K)$ is faithful (see Remark 3.5 in [6]).

An irreducible monomial character (respectively strongly monomial character) $\chi$ of $G$ is a character of the form $\chi=\psi^{G}$ (the induced character of $\psi$ ) for $\psi \in \operatorname{Lin}(H, K)$ (the set of linear characters of $H$ with kernel $K$ ) and some Shoda (respectively strong Shoda) pair $(H, K)$ of $G$. A finite group $G$ is monomial if every irreducible character of $G$ is monomial and it is strongly monomial if every irreducible character of $G$ is strongly monomial. It is well known that every 
abelian-by-supersolvable finite group is monomial. In [6] it is also proved that an abelian-by-supersolvable group is strongly monomial. Hence one obtains the following result, which again is fundamental in the proof of our main result.

Theorem 1.2 (Olivieri, del Río and Simón [6]). Let $G$ be a strongly monomial finite group (for example, an abelian-by-supersolvable group). Then, the primitive central idempotents of $\mathbb{Q} G$ are all the elements of the form $e(G, H, K)$, where $(H, K)$ is a strong Shoda pair of subgroups of $G$.

Assume, moreover, that $G$ is metabelian and let $A$ be a maximal abelian normal subgroup of $G$ containing the commutator subgroup $G^{\prime}$. Then, the primitive central idempotents are the elements of the form e $(G, H, K)$ with $(H, K)$ a pair of subgroups (with $K \subseteq H$ ) satisfying the following conditions:

(1) $K \unlhd H$ and $A \subseteq H$,

(2) $H / K$ is cyclic,

(3) if $H_{1}$ is a subgroup of $G$ so that $H \subseteq H_{1}$ and $H_{1} / K$ is abelian, then $H=H_{1}$. Moreover, if these properties are satisfied, then $(H, K)$ is a strong Shoda pair.

So, for abelian-by-supersolvable finite groups $G$ a character-free method has been given to compute the Wedderburn decomposition of the rational group algebra $\mathbb{Q} G$. In [7 an algorithmic method is presented to compute the Wedderburn decomposition of $k G$ for $G$ an arbitrary finite group and $k$ an arbitrary field of characteristic 0 using the Brauer-Witt theorem. This algorithm also has been implemented for the system GAP (package wedderga).

\section{Generic constructions of Central units}

The description of primitive central idempotents allows us to compute for strongly monomial finite groups $G$ a finite set of generators $B$ for a subgroup of finite index in $\mathcal{Z}(\mathcal{U}(\mathbb{Z} G))$, the group of central units of $\mathbb{Z} G$.

In order to do so, we consider $\mathbb{Z} G$ as a $\mathbb{Z}$-order in the rational group algebra $\mathbb{Q} G$; that is, the ring $\mathbb{Z} G$ is a finitely generated additive abelian group that contains a $\mathbb{Q}$-basis of the semisimple ring $\mathbb{Q} G$. Clearly, $\mathbb{Z} G \subseteq M=\bigoplus_{e} \mathbb{Z} G e$, where $e$ runs through all primitive central idempotents of $\mathbb{Q} G$. As $M$ is also a $\mathbb{Z}$-order in $\mathbb{Q} G$, we know that the finitely generated group $\mathcal{Z}(\mathcal{U}(\mathbb{Z} G))$ is of finite index in $\mathcal{Z}(\mathcal{U}(M))$. Hence, to prove that the finite set $B$ of $\mathcal{Z}(\mathcal{U}(\mathbb{Z} G))$ generates a subgroup of finite index in $\mathcal{Z}(\mathcal{U}(\mathbb{Z} G))$ it is sufficient (and necessary) to show that for every primitive central idempotent $e$ of $\mathbb{Q} G$ the group $\langle B\rangle$ contains a subgroup of finite index in the group of units of the form $(1-e)+u e$, where $u \in \mathcal{Z}(\mathcal{U}(\mathcal{O}))$ and $\mathcal{O}$ is a $\mathbb{Z}$-order in the center of $\mathbb{Q} G e$.

Furthermore, if $R_{1}$ and $R_{2}$ are $\mathbb{Z}$-orders in $\mathbb{Q} G$, then $\mathcal{U}\left(R_{1}\right)$ and $\mathcal{U}\left(R_{2}\right)$ are commensurable; i.e., $\mathcal{U}\left(R_{1}\right) \cap \mathcal{U}\left(R_{2}\right)$ is of finite index in both $\mathcal{U}\left(R_{1}\right)$ and $\mathcal{U}\left(R_{2}\right)$. Hence there exists a positive integer $m$ so that $u^{m} \in \mathcal{U}\left(R_{2}\right)$ for any $u \in \mathcal{U}\left(R_{1}\right)$. In particular, if $e$ is a central idempotent of $\mathbb{Q} G$, then there is a positive integer $m$ so that $(1-e)+u^{m} e=((1-e)+u e)^{m} \in \mathcal{U}(\mathbb{Z} G)$ for any $u \in \mathcal{U}(\mathbb{Z} G)$. Also, if $H$ and $K$ are subgroups of $G$ with $K$ normal in $H$, then $\hat{K}$ is a central idempotent in $\mathbb{Q} H$. Hence, there exists a positive integer $n$ so that $(1-\hat{K})+v^{n} \hat{K}=((1-\hat{K})+v \hat{K})^{n} \in$ $\mathcal{U}(\mathbb{Z} H) \subseteq \mathcal{U}(\mathbb{Z} G)$ for any $v \in \mathcal{U}(\mathbb{Z} H)$.

The reader is referred to [9, Chapter 1, Section 7] for the quoted statements on units of orders. 
At several points in the following proof we will need to recall that in a finitely generated abelian group, replacing generators by powers of themselves yields a subgroup of finite index.

Theorem 2.1. Let $G$ be a finite group that is strongly monomial. Let $B$ be the finite set consisting of elements of the form

$$
1+\sum_{t \in T}\left(\left(\left(\prod_{x \in N_{G}(K)}\left(b^{x}\right)^{m}\right)-1\right) \varepsilon(H, K)\right)^{t},
$$

where $(H, K)$ is a strong Shoda pair in $G, T$ is a right transversal for $N_{G}(K)$ in $G, b$ a Bass cyclic unit in $\mathbb{Z} H$ and $m=m(G, H, K)$ a positive integer so that $1+\left(\left(b^{x}\right)^{m}-1\right) e(G, H, K) \in \mathbb{Z} G$ for all $b$. Then $\langle B\rangle$ is of finite index in $\mathcal{Z}(\mathcal{U}(\mathbb{Z} G))$.

Proof. Let $(H, K)$ be a strong Shoda pair in $G$ and let $e=e(G, H, K)$ be the associated central primitive idempotent in $\mathbb{Q} G$. Since $H / K$ is a finite cyclic group, we know that the Bass cyclic units of $\mathbb{Z}(H / K)$ generate a subgroup of finite index in the finitely generated abelian group $\mathcal{U}(\mathbb{Z}(H / K))$. It is also well known and easy to verify that (some power of) such a Bass cyclic unit is a (natural) image of a Bass cyclic unit of $\mathbb{Z} H$. As $\varepsilon(H, K)$ is a primitive (central) idempotent of $\mathbb{Q}(H / K)$ we thus have that $\langle 1+(b-1) \varepsilon(H, K)| b$ a Bass cyclic unit of $\mathbb{Z} H\rangle$ is a subgroup of finite index in $\mathcal{U}(\mathbb{Z}(1-\varepsilon(H, K))+\mathbb{Z}(H / K) \varepsilon(H, K))$.

Let $N=N_{G}(K)$. Conjugation induces a natural action of $N$ on $\mathbb{Q} H$. For $\alpha \in \mathbb{Q} H$ and $g \in N$ we denote $g^{-1} \alpha g$ by $\alpha^{g}$. This action, in its turn, induces a natural action of $N / H$ on the field $\mathbb{Q} H \varepsilon(H, K) \cong \mathbb{Q}\left(\xi_{k}\right)$. From Proposition 1.1 we know that this is a faithful action. Therefore the centre of $\mathbb{Q} N \varepsilon(H, K)=$ $\mathbb{Q}\left(\xi_{k}\right) *_{\tau}^{\sigma} N / H$ is the fixed ring $(\mathbb{Q} H \varepsilon(H, K))^{N / H}$ of $\mathbb{Q} H \varepsilon(H, K)$ under this action. Now $(\mathbb{Z} H \varepsilon(H, K))^{N / H}$ is a commutative ring and a $\mathbb{Z}$-order in the field $(\mathbb{Q} H \varepsilon(H, K))^{N / H}$. Hence, its unit group is finitely generated, and thus it is readily verified that $\mathcal{U}\left((\mathbb{Z} H \varepsilon(H, K))^{N / H}\right)$ has $\left\{\prod_{g \in N} u^{g} \mid u \in \mathcal{U}(\mathbb{Z} H \varepsilon(H, K))\right\}$ as a subgroup of finite index. So, from the above we get that the units of the form

$$
\left(\prod_{g \in N} b^{g}\right) \varepsilon(H, K)
$$

with $b$ a Bass cyclic unit in $\mathbb{Z} H$, generate a subgroup of finite index in the unit group of an order in the centre of $\mathbb{Q} N \varepsilon(H, K)$. Because different $(\varepsilon(H, K))^{t}$, with $t \in T$, are orthogonal idempotents, elements of the form

$$
\sum_{t \in T}\left(\prod_{g \in N} b^{g} \epsilon(H, K)\right)^{t}
$$

are units in $\mathbb{Z} G e$.

From Proposition 1.1 we know that the central elements of $\mathbb{Q} G e(G, H, K)$ are the elements of the form $\sum_{t \in T} \alpha^{t}$, where $\alpha$ belongs to the centre of $\mathbb{Q} N \varepsilon(H, K)$. Hence, from the above we obtain that the elements of the form

$$
u=\sum_{t \in T}\left(\prod_{g \in N} b^{g} \varepsilon(H, K)\right)^{t}
$$


generate a subgroup of finite index in the unit group of a $\mathbb{Z}$-order in the center of $\mathbb{Q} G e(G, H, K)$. Of course, all elements $u^{m}$ then also generate a subgroup of finite index in such an order. Because distinct conjugates of $\varepsilon(H, K)$ are orthogonal, we clearly have

$$
u^{m}=\sum_{t \in T}\left(\prod_{g \in N}\left(b^{g}\right)^{m} \varepsilon(H, K)\right)^{t} .
$$

Because $e=\sum_{t \in T} \varepsilon(H, K)^{t}$, we get that

$$
1-e(G, H, K)+u^{m}=1+\sum_{t \in T}\left(\left(\left(\prod_{g \in N}\left(b^{g}\right)^{m}\right)-1\right) \varepsilon(H, K)\right)^{t} .
$$

Because of the assumption on $m$, we also know that these elements belong to $\mathbb{Z} G$ and they generate a subgroup of finite index in the unit group of the center of $\mathbb{Z}+\mathbb{Z} G e(G, H, K)$, a $\mathbb{Z}$-order in $\mathbb{Q}+\mathbb{Q} G e(G, H, K)$. This proves that the central units as described in the statement of the theorem generate a subgroup of finite index in the group of central units of $\mathbb{Z} G$.

Let $G$ be a finite group and $(H, K)$ a strong Shoda pair in $G$. For a Bass cyclic unit $b \in \mathbb{Z} H$ we denote by

$$
b^{(H, K)}=\prod_{g \in G}(1-\varepsilon(H, K)+b \varepsilon(H, K))^{g} .
$$

Note that, with notation as in Theorem 2.1

$$
(\epsilon(H, K)-b \epsilon(H, K))^{t}(\epsilon(H, K)-b \epsilon(H, K))^{t^{\prime}}=0
$$

for distinct elements $t, t^{\prime} \in T$ and a Bass cyclic unit $b \in \mathbb{Z} H$. Hence all elements

$$
(1-\varepsilon(H, K)+b \varepsilon(H, K))^{t},
$$

with $t \in T$, commute amongst themselves. Also, since $\mathbb{Z} H \widehat{K} \cong \mathbb{Z}(H / K)$ is commutative, elements of the form $(1-\varepsilon(H, K)+b \varepsilon(H, K))^{x}$ with $x \in N_{G}(K)$ commute amongst themselves. Hence, the factors defining $b^{(H, K)}$ commute and thus the definition is independent of the order of the factors. Note also that

$$
\prod_{x \in N_{G}(K)}(1-\varepsilon(H, K)+b \varepsilon(H, K))^{x}=1-\varepsilon(H, K)+\left(\prod_{x \in N_{G}(K)} b^{x}\right) \varepsilon(H, K) .
$$

It follows from the orthogonality of the various $\epsilon(H, K)^{t}$ and the above that generators defined in the previous theorem (as a summation of conjugates) can be rewritten as $\left(b^{(H, K)}\right)^{m(G, H, K)}$. Hence we obtain at once the following corollary.

Corollary 2.2. Let $G$ be a finite group that is strongly monomial (for example, $G$ is an abelian-by-supersolvable finite group). Then the group generated by all units 
of the form

$$
\begin{aligned}
& \left(b^{(H, K)}\right)^{m(G, H, K)} \\
& =\prod_{g \in G}\left(1-\varepsilon(H, K)+b^{m(G, H, K)} \varepsilon(H, K)\right)^{g} \\
& =\prod_{g \in T}\left(1-\varepsilon(H, K)+\left(\prod_{x \in N_{G}(K)}\left(b^{x}\right)^{m(G, H, K)}\right) \varepsilon(H, K)\right)^{g}
\end{aligned}
$$

with $(H, K)$ a strong Shoda pair, $b$ a Bass cyclic unit in $\mathbb{Z} H$ and $m(G, H, K)$ a positive integer so that $1-\varepsilon(H, K)+b^{m(G, H, K)} \varepsilon(H, K) \in \mathbb{Z} G$ for all Bass cyclic units $b \in \mathbb{Z} H$, is of finite index in $\mathcal{Z}(\mathcal{U}(\mathbb{Z} G))$.

Note that different strong Shoda pairs $(H, K)$ may give the same primitive central idempotent $e(G, H, K)$. It follows from the proofs that in the statement of Theorem 2.1 and Corollary 2.2 one only has to consider one strong Shoda pair (and the associated units) for each primitive central idempotent of $\mathbb{Q} G$.

We can "simplify" the proposed generators in case the primitive central idempotents of $\mathbb{Q} G$ are determined by strong Shoda pairs $(H, K)$ with $H$ normal in $G$. The latter occurs (see Theorem 1.2) for example if $G$ is a finite metabelian group.

Corollary 2.3. Let $G$ be a finite metabelian group. Then the group generated by all units of the form

$$
\prod_{g \in G}\left(1-\widehat{K}+b^{n(K)} \widehat{K}\right)^{g}
$$

with $(H, K)$ a strong Shoda pair and $H$ normal in $G, b$ a Bass cyclic unit in $\mathbb{Z} H$ and $n(K)$ the smallest positive integer so that $1-\widehat{K}+b^{n(K)} \widehat{K} \in \mathbb{Z} H$, is a central subgroup of finite index in $\mathcal{Z}(\mathcal{U}(\mathbb{Z} G))$.

Proof. Because $G$ is a metabelian finite group, Theorem 1.2 yields that all the primitive central idempotents of $\mathbb{Q} G$ are of the form $e(G, H, K)$ with $(H, K)$ a strong Shoda pair and $H$ normal in $G$. Clearly $\varepsilon(H, K)^{g}=\varepsilon\left(H, K^{g}\right) \in \mathbb{Q} H \widehat{K^{g}} \cong$ $\mathbb{Q}\left(H / K^{g}\right)$, a commutative semisimple ring that is in the centre of $\mathbb{Q} H$. Hence, with notation as in Corollary 2.2 all elements $\left(1-\varepsilon(H, K)+b^{m(G, H, K)} \varepsilon(H, K)\right)^{g}$ are central in $\mathbb{Q} H$.

Also all elements of the form $(1-\widehat{K}+b \widehat{K})^{g}$ (with $b$ a Bass cyclic unit in $\mathbb{Z} H$ ) are central in $\mathbb{Q} H$. Hence the order of any product of the factors mentioned in the statement of the corollary is irrelevant.

Because $H / K$ is cyclic, we know that the Bass cyclic units of $\mathbb{Z}(H / K)$ generate a subgroup of finite index in the abelian group $\mathcal{U}(\mathbb{Z}(H / K))$. As $\mathbb{Z}(H / K)$ is a $\mathbb{Z}$-order in $\mathbb{Q}(H / K) \cong \mathbb{Q} H \widehat{K}$, we thus obtain that the group generated by elements of the form $1-\widehat{K}+b \widehat{K}$ (with $b$ a Bass cyclic unit in $\mathbb{Z} H$ ) contains a subgroup of finite index $\operatorname{in} \mathcal{U}(\mathbb{Z}(1-\widehat{K})+\mathbb{Z} H \widehat{K})$. Hence, as $1-\varepsilon(H, K)+b \varepsilon(H, K)$ is a unit in $\mathbb{Z}(1-\widehat{K})+\mathbb{Z} H \widehat{K}$, we obtain that $1-\varepsilon(H, K)+b \varepsilon(H, K) \in\langle 1-\widehat{K}+b \widehat{K}| b$ a Bass cyclic unit in $\mathbb{Z} H\rangle$. Because of the commuting of the elements mentioned above, we obtain that

$$
\left.\left(b^{(H, K)}\right)^{m(G, H, K)} \in\left\langle\prod_{g \in G}(1-\widehat{K}+b \widehat{K})^{g}\right| b \text { a Bass cyclic unit in } \mathbb{Z} H\right\rangle .
$$


By Corollary 2.2 the group generated by all $\left(b^{(H, K)}\right)^{m(G, H, K)}$ is of finite index in the centre of $\mathcal{U}(\mathbb{Z} G)$. Because of the comment given before the corollary, the result thus follows.

\section{REDUCTION MACHINERY}

In this section, we prove a general result that yields generators for central units of $\mathbb{Z} G$ from subgroups and quotient groups of $G$. An application is given for solvable Frobenius groups.

Theorem 3.1. Let $G$ be a finite group with normal subgroups $N$ and $M$ so that $N \subseteq M$. Suppose the following conditions hold:

(1) $\left\langle\overline{c_{1}}, \ldots, \overline{c_{n}}\right\rangle$ is a subgroup of finite index in $\mathcal{Z}(\mathcal{U}(\mathbb{Z}(G / N)))$,

(2) $\left\langle d_{1}, \ldots, d_{m}\right\rangle$ is a subgroup of finite index in $\mathcal{Z}(\mathcal{U}(\mathbb{Z} M))$.

If $\mathcal{Z}(\mathcal{U}(\mathbb{Z} G)) \cap(1+\Delta(G, N)) \subseteq \mathbb{Z} M$, then the group

$$
\left\langle 1-\widehat{N}+c_{i}^{e(N)} \widehat{N}, \prod_{g \in G} d_{j}^{g} \mid 1 \leq i \leq n, 1 \leq j \leq m\right\rangle
$$

(with e $(n)$ the smallest positive integer so that $1-\widehat{N}+c_{i}^{e(N)} N \in \mathbb{Z} G$ ) is a subgroup of finite index in $\mathcal{Z}(\mathcal{U}(\mathbb{Z} G))$.

Proof. It is easy to see that $1-\widehat{N}+c_{i}^{e(N)} \widehat{N}$ is a central unit in $\mathbb{Z} G$. Since $\left\langle\bar{c}_{1} e(N), \ldots,{\overline{c_{n}}}^{e(N)}\right\rangle$ is of finite index in $\mathcal{Z}(\mathcal{U}(\mathbb{Z}(G / N))$, it follows that for any central unit $z \in \mathcal{U}(\mathbb{Z} G)$ there exists a positive integer $w$ and a central unit $v \in 1+\Delta(G, N)$ so that $z^{w} v \in\left\langle 1-\widehat{N}+c_{i}^{e(N)} \widehat{N} \mid 1 \leq i \leq n\right\rangle$. Now, $v$ clearly is a central unit in $\mathbb{Z} M$. Hence, by assumption, $v^{r} \in\left\langle d_{i} \mid 1 \leq i \leq m\right\rangle$, where $r$ is the index of $\left\langle d_{1}, \ldots, d_{m}\right\rangle$ in $\mathcal{U}(\mathbb{Z} M)$. Since all conjugates $d_{i}^{g}$ and all $d_{j}$ belong to $\mathcal{Z}(\mathcal{U}(\mathbb{Z} M)$ ) (and thus commute with each other), it follows that $v^{r|G|} \in\left\langle\prod_{g \in G} d_{j}^{g} \mid 1 \leq j \leq m\right\rangle$. Hence $z^{w r|G|}$ belongs to the finitely generated group described in the statement. So the result follows.

The following lemma gives a sufficient condition for the third property in the previous theorem to be satisfied.

Lemma 3.2. Let $G$ be a finite group with normal subgroup $N$. Assume that $x \in G$ has the property that $C_{N}(x)=\{1\}$. Then

(1) $x$ is conjugate to $x n$ for all $n \in N$,

(2) $x$ cannot appear in the support of any central unit of $\mathbb{Z} G$ which also belongs to $1+\Delta(G, N)$.

Proof. We are given that $\left\{n x n^{-1} \mid n \in N\right\}$ contains $|N|$ distinct elements. Since $n x n^{-1}=x\left(x^{-1} n x n^{-1}\right)$ and $x^{-1} n x n^{-1} \in N$, the first statement follows.

For the second, assume $\alpha$ is a central unit of $\mathbb{Z} G$ lying in $1+\Delta(G, N)$. Since $\alpha$ is central in $\mathbb{Z} G$, we have that

$$
\alpha=a_{1} C_{1}+a_{2} C_{2}+\cdots+a_{t} C_{t},
$$

where $a_{i} \in \mathbb{Z}$ and $C_{i}$ is a conjugacy class sum for each $i$. Since $\alpha \in 1+\Delta(G, N)$, we also have that

$$
\alpha=1+\sum g_{i} \alpha_{i}
$$

where the $g_{i}$ belong to a transversal of $N$ in $G$ and $\alpha_{i} \in \Delta(N)$ for each $i$. It follows from the first part of the result that the conjugacy class sum $C_{j}$ of $x$ must be of the 
form $\beta \widehat{N}$, where $\beta$ is a $\mathbb{Z}$-linear sum of transversal elements of $N$ in $G$. Since each such transversal element appears in the support of a unique term $g_{i} \alpha_{i}$, we must have, for this $C_{j}$,

for certain terms $g_{i} \alpha_{i}$. But

$$
a_{j} C_{j}=\sum g_{k} \alpha_{k}
$$

$$
a_{j} C_{j} \widehat{N}=a_{j} \beta \widehat{N} \widehat{N}=a_{j} \beta \widehat{N}
$$

while

$$
\sum g_{k} \alpha_{k} \widehat{N}=0
$$

Hence it follows that $a_{j}=0$ for such conjugacy class sums, and we are done.

Corollary 3.3. Let $G$ be a finite group. If $G$ is a Frobenius group with kernel $N$, and $|G / N|$ is odd, then the following elements generate a subgroup of finite index in $\mathcal{Z}(\mathcal{U}(\mathbb{Z} G))$ :

$$
\prod_{g \in G}\left(1-\widehat{K}+b^{e(K)} \widehat{K}\right)^{g} \quad \text { and } \quad \prod_{g \in G} c^{g},
$$

with $K$ a normal subgroup of $G$ so that $N \subseteq K$, and $(H, K)$ a strong Shoda pair, $b$ a Bass cyclic unit in $\mathbb{Z} H$, and $c$ a central unit of $\mathbb{Z} N$ as described in the statement of Corollary 2.2.

Proof. The given conditions tell us that the group $G / N$ is metacyclic. Hence, by Corollary 2.3 . we know that the generators of the type $\prod_{g \in G}\left(1-\widehat{K}+b^{e(K)} \widehat{K}\right)^{g}$ generate a subgroup of finite index in $1-\widehat{N}+Z G \widehat{N}$. Clearly, because $G$ is Frobenius, $C_{N}(x)=\{1\}$ for any $x \in G \backslash N$. Hence, by Lemma 3.2, the central units of $\mathbb{Z} G$ that belong to $1+\Delta(G, N)$ must lie in $\mathbb{Z} N$. Since $N$ is nilpotent, the result follows from Theorem 3.1

Note that because of a result mentioned in the introduction [4, the units $c$ used in the above corollary may also be replaced by $b_{(n)}$, where $n$ is the nilpotency class of $N$ and $b$ is a Bass cyclic unit in $\mathbb{Z} N$.

\section{ACKNOWLEDGEMENT}

The authors would like to thank the referee for several helpful observations and suggestions.

\section{REFERENCES}

1. H. Bass, The Dirichlet unit theorem, induced characters and Whitehead groups of finite groups, Topology 4 (1966), 391-410. MR0193120 (33:1341)

2. R. A. Ferraz, J.J. Simón, Central units in metacyclic integral group rings, Comm. Algebra 36 (2008), no. 10, 3708-3722. MR2458401(2009h:20006)

3. E. Jespers, A. Giambruno, Central idempotents and units in rational group algebras of alternating groups, International Journal of Algebra and Computation 8 (1998), Issue 4, 467-477. MR:1663562(99m:16048)

4. E. Jespers, M. Parmenter, S.K. Sehgal, Central units of integral group rings of nilpotent groups, Proc. Amer. Math. Soc. 124 (1996), no. 4, 1007-1012. MR1328353 (96g:16044)

5. Y. Li, M.M. Parmenter, Central units of the integral group ring $Z A_{5}$, Proc. Amer. Math. Soc. 125 (1997), no. 1, 61-65. MR 1353390 (97c:16041)

6. A. Olivieri, Á. del Río, J.J. Simón, On monomial characters and central idempotents of rational group algebras, Comm. Algebra 32 (2004), no. 4, 1531-1550. MR 2100373(2005i:16054)

7. G. Olteanu, Computing the Wedderburn decomposition of group algebras by the Brauer-Witt theorem, Math. Comp. 76 (2007), no. 258, 1073-1087. MR2291851(2008b:16036) 
8. J. Ritter and S.K. Sehgal, Units of group rings of solvable and Frobenius groups over large rings of cyclotomic integers, Journal of Algebra 158 (1993), 116-129. MR1223670|(95d:16045)

9. S. K. Sehgal, Units in integral group rings, Longman Scientific and Technical, Essex, 1993. MR:1242557 (94m:16039)

Department of Mathematics, Vrije Universiteit Brussel, Pleinlaan 2, 1050 Brussel, BELGIUM

E-mail address: ef jesper@vub.ac.be

Department of Mathematics and Statistics, Memorial University of Newfoundland, St. John's, Newfoundland, A1C 5S7, Canada

E-mail address: mparmen@mun.ca 